\title{
Aprimoramento de Modelos Subótimos para Identificação de Sistemas
}

\author{
Pedro Augusto da Silva Braga* \\ Samir Angelo Milani Martins ** \\ * Programa de Pós-Graduação em Engenharia Elétrica, Universidade \\ Federal de São João del-Rei, MG, (e-mail: pedro.asb@hotmail.com). \\ ** Departamento de Engenharia Elétrica, Universidade Federal de São \\ João del-Rei, MG (e-mail: martins@ufsj.edu.br)
}

\begin{abstract}
One of the stages of the identification of systems is the determination of the structure of the model, in which two techniques are generally employed: the Error Reduction Rate (ERR) and the Akaike Information Criteria (AIC). However, these are of a mono-objective character, which leads them to have limitations, since they consider only the dynamic data of the system. As a result, this article proposes a technique that seeks to improve certain behaviors of the identified model, such as static. In this context, it was applied in a polynomial NARMAX model of a system composed of a DC-DC buck converter, with the intention of reducing the static and dynamic errors of the model. As a result, it was possible to obtain a set of efficient solutions.

Resumo: Uma das etapas da identificação de sistemas é a determinação da estrutura do modelo, na qual são geralmente empregadas duas técnicas: a Taxa de Redução de Erro (ERR) e o Critério de Informação de Akaike (AIC). Todavia, estas são de caráter mono-objetivo, o que as leva a terem limitações, uma vez que consideram apenas os dados dinâmicos do sistema. Em virtude disto, este artigo propõe uma técnica que busca aprimorar determinados comportamentos do modelo identificado, como, por exemplo, o estático. Nesse contexto, ela foi aplicada em um modelo NARMAX polinomial de um sistema composto por um Conversor CC-CC Buck, com a intenção de diminuir os erros estático e dinâmico do modelo. Como resultado, foi possível obter um conjunto de soluções eficientes.
\end{abstract}

Keywords: NARMAX model; model enhancement; multi-objective system identification; static and dynamic behavior; nonlinear dynamics.

Palavras-chaves: Modelo NARMAX; aprimoramento do modelo; identificação de sistemas multi-objetivo; comportamento estático e dinâmico; dinâmica não linear.

\section{INTRODUÇÃO}

A identificação de sistemas é uma área da ciência que estuda formas de representar um sistema, em que pouco ou, até mesmo, nenhum conhecimento prévio do sistema seja necessário. Diante disso, pode ser considerada uma arte da construção de modelos matemáticos a partir dos dados de entrada-saída (Huang et al., 2018). Ademais, vale salientar que um modelo matemático pode ser entendido como uma abstração de um sistema real representado por meio de equações (Aguirre, 2007).

As técnicas de identificação de sistemas estão presentes nas mais variadas áreas, como, por exemplo, na economia: previsão de índices inflacionários (Ghosh et al., 2018; Gupta et al., 2015); na engenharia: identificação de turbina a gás de aeronave (Chiras et al., 2001); na área social: em análise de fatores associados às variações temporais nas fatalidade no trânsito (Sukhai et al., 2011); nos sistemas biomédicos: identificação do sistema cardiovascular humano (Irigoyen e Miñano, 2013); entre outras áreas. Além disso, existem muitos estudos sendo realizados, a fim de aprimorarem os procedimentos de identificação como: a utilização de modelos polinomiais para descrever o comportamento de histerese (Martins e Aguirre, 2016); técnica para aumentar a confiabilidade numérica na simulação livre de modelos polinomiais NARMAX (Nonlinear AutoRegressive Moving Average model with eXogenous input) (Nepomuceno e Martins, 2016); e a identificação de sistemas dinâmicos com Redes Neurais Convolucionais (CNNs - do inglês Convolutional Neural Networks) (Genc, 2017).

Entre as representações matemáticas para modelar sistemas, a NARMAX polinomial é uma que possui baixa complexidade e permite incorporar informações (ganho estático, número e localização de pontos fixos e a função estática) que se tem a priori do sistema (Nepomuceno et al., 2007). Em decorrência disso, o modelo poderá ser melhorado de acordo com o acréscimo dessas informações, como, por exemplo na sua representatividade do comportamento estático.

Uma das etapas da identificação de sistemas é a detecção de estrutura, em que duas técnicas são geralmente empregadas. A primeira é a Taxa de Redução de Erro (ERR - do inglês Error Reduction Ratio) (Billings, 1980), a qual visa 
obter os regressores, de modo hierárquico, que minimizem a variância normalizada dos dados dinâmicos do sistema; e a segunda é o Critério de Informação de Akaike (AIC - do inglês Akaike Information Criteria) (Akaike, 1974) que é utilizado para determinar o número de regressores que serão incluídos no modelo. No entanto, essas técnicas apresentam limitações por serem de caráter mono-objetivo e, por isso, não ajudam a explicar outros comportamentos do sistema, como, por exemplo, o estático (Besharati et al, 2016).

Diante desse impasse, o objetivo deste trabalho é propor um procedimento que, a partir de um modelo obtido por um método subótimo qualquer, como, por exemplo, por ERR e AIC, seja possível obter melhores modelos, os quais levam em conta também aspectos ignorados durante a obtenção desse modelo (ganho estático, dimensão fractal, expoente de Lyapunov e entre outros). Nesse sentido, o procedimento foi aplicado em um modelo NARMAX identificado por métodos de aspectos mono-objetivo de um sistema composto por um conversor CC-CC buck, a técnica foi aplicada com a intenção de diminuir os erros estático e dinâmico. A metodologia abordada possibilitou encontrar um conjunto de soluções eficientes, ou seja, modelos que conseguem explicar mais satisfatoriamente os comportamentos avaliados.

A estrutura do artigo está ordenada da seguinte forma: nesta seção contém uma breve introdução sobre identificação de sistemas com algumas técnicas empregadas na área, além do objetivo do trabalho. Ademais, os conceitos preliminares para o entendimento do trabalho desenvolvido, a metodologia utilizada, assim como os resultados estão, respectivamente, nas seções 2,3 e 4 . Por fim, a conclusão contendo algumas sugestões de possíveis trabalhos futuros estão na seção 5 .

\section{CONCEITOS PRELIMINARES}

\subsection{Identificação de Sistemas}

Para realizar a identificação de um sistema, são realizadas cinco etapas fundamentais (Ljung, 1987):

As cinco etapas fundamentais para realizar a identificação de um sistema são (Ljung, 1987):

(1) Teste dinâmico e coleta de dados: o primeiro passo consiste em coletar dados do sistema a ser identificado. Para sistemas físicos, é primordial que o sinal de excitação do sistema seja bem projetado, assim como a frequência de amostragem dos dados coletados.

(2) Escolha da representação matemática: esta é importante pelo fato de interferir diretamente na qualidade do modelo obtido. Existem diversos tipos de representações matemáticas, como, por exemplo: NARMAX polinomial, RBF (Radial Basis Functions), ANN (Artificial Neural Networks), NFN (NeoFuzzy Neuron), entre outras.

(3) Determinação da estrutura do modelo: conforme já foi apresentado, os regressores que irão compor o modelo são determinados nessa etapa.

(4) Estimação dos Parâmetros: os parâmetros estimados têm por função ponderar a contribuição necessária de cada regressor para representar um determinado sis- tema. Nesse trabalho os parâmetros foram estimados via Mínimos Quadrados Estendidos (MQE).

(5) Validação do modelo: em relação às técnicas de validação do modelo, existem diferentes tipos e o emprego delas varia de acordo com a aplicação do modelo.

Nesse estudo, as técnicas de validação foram aplicadas na predição livre do modelo e as características dinâmicas e estáticas do modelo foram analisadas. O primeiro índice utilizado é o RMSE, do inglês Root Mean Squared Error, o qual quantificou as predições, expresso pela Equação (1):

$$
R M S E=\frac{\sqrt{\sum_{k=1}^{n}(y(k)-\hat{y}(k))^{2}}}{\sqrt{\sum_{k=1}^{n}(y(k)-\bar{y})^{2}}},
$$

sendo $\hat{y}$ a simulação livre do sinal para o caso dinâmico e o valor estático de saída para o caso estático e $\bar{y}$ é o valor médio do sinal medido $y(k)$. Esse índice mede o erro em uma unidade de medida coerente com os dados reais (Nepomuceno et al., 2007). Nesse contexto, modelos bons, comumente, são aqueles que apresentam esse índice normalizado com o valor menor que a unidade (Martins et al., 2012). Quando isso ocorre, significa que, em média, o erro demonstrado pelo modelo é menor que o erro dado pela média da série temporal.

Outra métrica de validação é o Somatório dos Erros Quadráticos Estático e Dinâmico (Nepomuceno et al., 2007), em que as Equações (2) e (3) os representam respectivamente.

$$
\begin{aligned}
& J_{e}=\left(y_{e}-\hat{y}_{e}\right)^{T}\left(y_{e}-\hat{y}_{e}\right), \\
& J_{d}=\left(y_{d}-\hat{y}_{d}\right)^{T}\left(y_{d}-\hat{y}_{d}\right),
\end{aligned}
$$

em que $y_{e}$ e $y_{d}$ são vetores de saída do sistema para os casos estático e dinâmico nessa ordem, já $\hat{y}_{e}$ é o vetor com os valores de saída para o caso estático e $\hat{y}_{d}$ é vetor com a simulação livre do sinal para o caso dinâmico.

\subsection{Modelos NARMAX polinomiais}

Os modelos NARMAX são modelos discretos no tempo que buscam explicar o valor da saída $y(k)$ em funções de valores anteriores da saída e de entrada, eles podem ser expressos por (Billings, 1980):

$$
\begin{aligned}
y(k)= & F^{\ell}\left[\left(y(k-1), \ldots, y\left(k-n_{y}\right),\right.\right. \\
& u\left(k-\tau_{d}\right), \ldots, u\left(k-n_{u}\right), \\
& \left.e(k-1), \ldots, e\left(k-n_{e}\right)\right]+e(k)
\end{aligned}
$$

em que $n_{y}, n_{u}$ e $n_{e}$ indicam os atrasos em $y, u$ e $e$ respectivamente, já $\tau_{d}$ é o atraso puro de tempo do sistema. A parcela $e(k)$ representa todos os efeitos que não foram possíveis de serem representados por $F^{\ell}[\cdot]$, uma função polinomial com grau de não linearidade $\ell \in \mathbb{N}$.

Para o caso em que a Equação (4) possuir o grau de não linearidade unitário $(\ell=1)$, a representação passa a se chamar ARMAX. A parte média móvel (MA) do modelo é inserida caso exista ruído autocorrelacionado (colorido) e o modelo inclua regressores da saída. Nessa situação, se o método dos Mínimos Quadrados (MQ) estimar o 
modelo, os resíduos apresentarão alguma dinâmica que não foi explicada pelo modelo. Em tal caso, o MQ se torna polarizado ou tendencioso. Em consequência disso, um outro método que não seja polarizado deverá ser aplicado, como o método dos Mínimos Quadrados Estendidos (MQE).

Outra representação possível para modelo polinomial NARMAX é expressa na Equação (5):

$$
\mathbf{y}=\Psi \hat{\boldsymbol{\theta}}+\boldsymbol{\xi},
$$

em que y indica o vetor com os dados de saídas, $\Psi$ é a matriz com os regressores, $\hat{\boldsymbol{\theta}}$ representa os parâmetros estimados, e $\boldsymbol{\xi}$ é a parte que não foi possível ser representada pelo modelo, no qual $\hat{\mathbf{y}}=\Psi \hat{\theta}$. A Figura 1 apresenta um exemplo de representação de um vetor de saída y de um sistema na base composta pelos regressores $\psi_{1}$ e $\psi_{2}$.

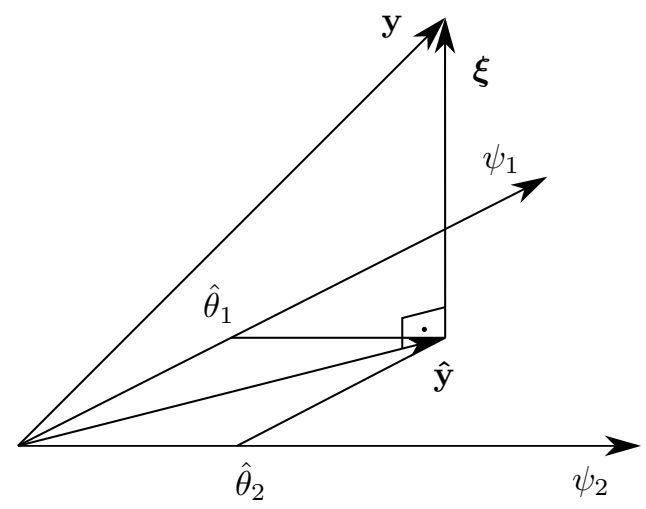

Figura 1. Base de regressores para compor um modelo estimado por um estimador ortogonal.

\subsection{Taxa de Redução de Erro - ERR}

A ERR é uma técnica que tem como base o erro dinâmico de predição um passo à frente. Desse modo, ela associa cada regressor candidato na composição do modelo a um índice correspondente à contribuição na explicação da variância dos dados de saída do sistema, sendo que esta é definida por:

$$
\operatorname{var}[\xi(k)]=\lim _{N \rightarrow \infty} \frac{1}{N}\left[y^{T} y-\sum_{i=1}^{N} g_{i}^{2} w_{i}^{T} w_{i}\right]
$$

em que $g_{i}$ indica cada elemento do vetor de parâmetros, $w_{i}$ o regressor ortogonal incluso (associado a um regressor $\psi_{i}$ candidato ao modelo), e $y$ representa a série temporal dos dados de saída de tamanho $N$.

Para cada termo incluído no modelo, temos como consequência a diminuição da variância de um fator de $\frac{1}{N}\left(g_{i}^{2} w_{i}^{T} w i\right)$. A ERR, devido à inclusão de um i-ésimo regressor, é definida por (Korenberg et al., 1988):

$$
[E R R]_{i}=\frac{\hat{g}_{i}^{2}\left\langle\mathbf{w}_{i}, \mathbf{w}_{i}\right\rangle}{\langle\mathbf{y}, \mathbf{y}\rangle},
$$

em que $\hat{g}_{i}$ se refere à estimativa dos parâmetros, $\mathbf{w}_{i}$ é o regressor ortogonal $i$ e $\mathbf{y}$ os dados de saída. Dessa maneira, quanto maior a $E R R$ de um determinado regressor, esperase um melhor modelo caso ele seja utilizado. Já em relação aos parâmetros, eles podem ser calculados pela seguinte forma:

$$
\hat{g}_{i}=\frac{\left\langle\mathbf{w}_{i}, \mathbf{y}\right\rangle}{\left\langle\mathbf{w}_{i}, \mathbf{w}_{i}\right\rangle}, \quad i=1, \ldots, n_{\theta},
$$

em que $n_{\theta}$ é o número total de regressores candidatos para compor o modelo. Maiores detalhes estão disponíveis em (Aguirre, 2007).

\subsection{Critério de Informação de Akaike - AIC}

Após determinar a ordem dos regressores mais importantes para a representação do sistema (classificação hierárquica dos termos) pelo ERR, é necessário definir o número que irá compor o modelo. Para isso, poderá ser utilizado o método do $\mathrm{AIC}$, de modo que represente o sistema de forma satisfatória. Esta técnica se baseia no número de termos do modelo que minimize a função de custo $J_{A I C}$ (Akaike, 1974):

$$
J_{A I C}=N \log (\operatorname{var}[\xi(k)])+2 n_{\theta},
$$

em que $N$ representa o tamanho da série temporal dos dados de identificação, $\operatorname{var}[\xi(k)]$ é a variância do erro de modelagem, ou seja, o erro de predição um passo à frente, e $n_{\theta}$ o número de regressores candidatos para compor o modelo.

\section{METODOLOGIA}

Dado um sistema identificado com 2 ou mais regressores, existe a possibilidade de que o número de regressores encontrados por alguma técnica, como, por exemplo, por AIC, seja superior à dimensão mínima necessária para uma base de regressores representar qualquer dado de saída do sistema, ou seja, maior que a dimensão do sistema modelado. Nessa circunstância, não haveria uma única representação do sistema na base de regressores, o que poderia conduzir a várias representações do sistema (estimativa de parâmetros diferentes), levando assim a uma estimativa sobreparametrizada do sistema. Além disso, haveria então ao menos um regressor espúrio, desnecessário para representação do sistema e que teria impacto negativo na composição do modelo final, aumentando assim o $\boldsymbol{\xi}$.

Diante desse fato, a técnica proposta no presente trabalho tem como objetivo estimar novos modelos retirando regressores que podem não ser necessários ou de pouca importância para compor a base de espaço do vetor de saída dinâmico e/ou estático, chegando assim em um conjunto de solução eficientes.

\subsection{Técnica para Melhoramento do Modelo}

O primeiro passo consiste em obter um modelo subótimo (nomeado nesse estudo como modelo nominal 1) utilizando alguma(s) técnica(s), como por exemplo, as utilizadas nesse trabalho (ERR e AIC). O segundo passo se resume em avaliar métricas de desempenho de acordo com a necessidade de quem está empregando o modelo, assim, 
a análise de cada regressor será feita, a fim de avaliar a importância na representação do sistema e verificar se este consiste ou não em um termo espúrio para aquela característica do sistema. Desse modo, novos modelos são gerados, retirando um regressor diferente em cada.

No presente caso, os comportamentos estático e dinâmico do sistema foram analisados, mas qualquer outra característica poderia ser analisada aqui sem perda de generalidade da técnica proposta. Sendo assim, foi avaliado nos novos modelos se algum consegue reduzir simultaneamente os erros estático e dinâmico em comparação com o modelo nominal 1, ou seja, é avaliado se a remoção de algum regressor gera modelos que suplantam o modelo nominal 1, isto é, modelos com menores RMSE estático/dinâmico e o somatório dos Erros Quadráticos Estático e Dinâmico. Caso exista algum, este será denominado por nominal 2 e o processo é repetido. Este sendo um processo iterativo, o ponto de parada é até não existir melhoras nos comportamentos avaliados juntamente em comparação com o modelo nominal $n$ da iteração $n$, ou seja, o processo termina quando retirado qualquer regressor de um modelo nominal $n$ não existem mais aperfeiçoamento nos comportamentos examinados.

Após terminar o processo descrito, será o momento de obter o conjunto de soluções eficientes. Nesse ponto, os modelos que melhor expliquem os comportamentos analisados serão avaliados, sendo traçado, no presente caso, a relação de $J_{e}$ por $J_{d}$, com os melhores modelos obtidos. Vale salientar ainda que a escolha do modelo a ser utilizado, no contexto das soluções eficientes, depende da necessidade de quem estiver utilizando-os.

\subsection{Sistema Identificado e Aplicação da Técnica}

O sistema experimental utilizado para fazer a identificação e as análises foi um conversor CC-CC buck. Os dados foram originalmente coletados em (Aguirre et al., 2000). O sinal de entrada aplicado no MOSFET do sistema foi um sinal binário pseudoaleatório (PRBS - do inglês Pseudo Random Binary Signal) variado entre os valores $2,2 \mathrm{~V}$ e $2,5 \mathrm{~V}$.

As informações de entrada e saída foram divididas em duas parcelas distintas com o mesmo número de amostras (84 cada), sendo a primeira para identificar e a segunda para validar o modelo. A escolha da representação matemática foi a NARMAX polinomial e os atrasos escolhidos foram: $n_{y}=2, n_{u}=2$ e $n_{e}=2$, com grau de não linearidade $\ell=2$. Ao utilizar a ERR, a ordem dos regressores mais importantes foram definidos e o número para formar o modelo definido por AIC.

Para o cálculo dos dados estáticos de um conversor, pela teoria de eletrônica de potência, a curva estática de um conversor CC-CC buck é dada pela Equação (10):

$$
\bar{y}=\frac{3}{4} v_{d}-\frac{v_{d}}{3} \bar{u}
$$

em que $\bar{y}$ é a tensão de saída; $v_{d}$, o valor da tensão CC de alimentação, nesse trabalho igual a 24 Volts; e $\bar{u}$, o valor da entrada em um estado estacionário. Para efeitos de validação do comportamento estático, a entrada varia de 0 a $5 \mathrm{~V}$, com um total de 51 pontos. A Equação (10) mostra que o comportamento estático do sistema é linear, mas em relação à dinâmica, é não-linear (Martins et al., 2012).

\section{RESULTADOS}

A metodologia apresentada foi aplicada na identificação do modelo nominal, chegando ao resultado conforme a Equação (11):

$$
\begin{aligned}
y_{\Lambda_{\mathrm{N}}}(k)= & 1,3299 y(k-1)-0,5648 y(k-2) \\
& +0,2568 y(k-1)^{2}+24,0242 u(k-1)^{2} \\
& +0,2285 y(k-2)^{2}-0,4548 y(k-2) y(k-1) \\
& +170,9235+0,7591 u(k-1) y(k-1) \\
& -125,4085 u(k-1)
\end{aligned}
$$

Como 9 regressores foram obtidos no primeiro modelo, o próximo passo foi estimar outros 9 modelos (iteração 1), retirando em cada caso um regressor diferente. Em seguida, para analisar a qualidade dos modelos, o RMSE foi calculado para o caso estático $\left(R M S E_{e}\right)$, bem como para o dinâmico $\left(R M S E_{d}\right)$; e os erros quadráticos estático e dinâmico, normalizados na faixa de 0 a 1 (as iterações 1 e 2 foram normalizadas na mesma faixa). As tabelas 1 e 2 apresentam os resultados para os casos estático e dinâmico, nessa ordem.

Tabela 1. Validação do comportamento estático.

\begin{tabular}{cccc} 
Modelo & Regressor Retirado & $R M S E_{e}$ & $J_{e}$ \\
\hline$\Lambda_{\mathrm{N}}$ & - & 4,96600 & 0,79983 \\
$\Lambda_{1}$ & $y(k-1)$ & 4,59496 & 0,68472 \\
$\Lambda_{2}$ & $y(k-2)$ & 5,33474 & 0,92307 \\
$\Lambda_{3}$ & $y(k-1)^{2}$ & 4,80620 & 0,74916 \\
$\Lambda_{4}$ & $u(k-1)^{2}$ & 0,86417 & 0,02387 \\
$\Lambda_{5}$ & $y(k-2)^{2}$ & 5,55250 & 1 \\
$\Lambda_{6}$ & $y(k-2) y(k-1)$ & 5,27576 & 0,90277 \\
$\Lambda_{7}$ & $C$ & 1,98378 & 0,12733 \\
$\Lambda_{8}$ & $u(k-1) y(k-1)$ & 4,11776 & 0,54981 \\
$\Lambda_{9}$ & $u(k-1)$ & 1,34381 & 0,05823 \\
\hline \multicolumn{4}{c}{}
\end{tabular}

Os resultados dos modelos para o comportamento estático mostram que a retirada de qualquer um dos regressores: $y(k-2), y(k-2)^{2}$ ou $y(k-2) y(k-1)$, todos estes contendo em comum a saída com atraso $2(y(k-2))$, acarreta uma piora do modelo do ponto de vista estático. Os regressores $u(k-1)^{2}, C$ e $u(k-1)$ não se mostraram essenciais para representarem o comportamento estático.

Tabela 2. Validação do comportamento dinâmico.

\begin{tabular}{cccc} 
Modelo & Regressor Retirado & $R M S E_{d}$ & $J_{d}$ \\
\hline$\Lambda_{\mathrm{N}}$ & - & 0,17875 & 0,00114 \\
$\Lambda_{1}$ & $y(k-1)$ & 0,19847 & 0,14977 \\
$\Lambda_{2}$ & $y(k-2)$ & 0,18795 & 0,06856 \\
$\Lambda_{3}$ & $y(k-1)^{2}$ & 0,26576 & 0,77402 \\
$\Lambda_{4}$ & $u(k-1)^{2}$ & 0,17859 & 0 \\
$\Lambda_{5}$ & $y(k-2)^{2}$ & 0,22993 & 0,41910 \\
$\Lambda_{6}$ & $y(k-2) y(k-1)$ & 0,23047 & 0,42404 \\
$\Lambda_{7}$ & $C$ & 0,18859 & 0,07332 \\
$\Lambda_{8}$ & $u(k-1) y(k-1)$ & 0,28074 & 0,93757 \\
$\Lambda_{9}$ & $u(k-1)$ & 0,18157 & 0,02142 \\
\hline
\end{tabular}


Em relação à representação do comportamento dinâmico do modelo, os regressores mais importantes são: $u(k-$ 1) $y(k-1), y(k-1)^{2}, y(k-2) y(k-1)$ e $y(k-2)^{2}$, caso algum destes seja retirado, há um significativo aumento no $R M S E_{d}$ e em $J_{d}$, o que significa que a ausência de um dos quatro causa uma piora na representação dinâmica do sistema, sendo essenciais para compor a base da representação dinâmica. Além disso, vale ressaltar que eles são não-lineares, justificando suas relevâncias pelo comportamento dinâmico do sistema ser também nãolinear.

A ausência do regressor $u(k-1)^{2}$ (modelo $\left.\Lambda_{4}\right)$ acarreta uma melhora pequena no comportamento dinâmico, além de contribuir positivamente no comportamento estático do sistema. Com base nisso, apesar de o regressor ser não-linear, ele não é essencial para compor a base da representação dinâmica, podendo ser considerado, assim, como espúrio. Para continuar a análise buscando melhores modelos, o processo de retirada individual de regressores para compor novos modelos foi feito a partir do modelo $\Lambda_{4}$ (iteração 2). Este é nomeado agora por $\Lambda_{\mathrm{N} 2}$, ou seja, modelo nominal 2. As tabelas 3 e 4 apresentam os resultados para os casos estático e dinâmico respectivamente.

Tabela 3. Validação do comportamento estático.

\begin{tabular}{cccc} 
Modelo & Regressor Retirado & $R M S E_{e}$ & $J_{e}$ \\
\hline $\boldsymbol{\Lambda}_{\mathbf{N 2}}$ & - & $\mathbf{0 , 8 6 4 1 7}$ & $\mathbf{0 , 0 2 3 8 7}$ \\
$\Lambda_{\mathbf{2 1}}$ & $y(k-1)$ & $\mathbf{0 , 7 5 8 9 2}$ & $\mathbf{0 , 0 1 8 3 2}$ \\
$\Lambda_{22}$ & $y(k-2)$ & 0,96694 & 0,02997 \\
$\Lambda_{23}$ & $y(k-1)^{2}$ & 0,91933 & 0,02706 \\
$\Lambda_{24}$ & $y(k-2)^{2}$ & 1,12384 & 0,04062 \\
$\Lambda_{25}$ & $y(k-2) y(k-1)$ & 1,06585 & 0,03650 \\
$\Lambda_{26}$ & $C$ & 0,27439 & 0,00208 \\
$\boldsymbol{\Lambda}_{\mathbf{2 7}}$ & $u(k-1) y(k-1)$ & $\mathbf{0 , 2 3 8 6 1}$ & $\mathbf{0 , 0 0 1 4 8}$ \\
$\boldsymbol{\Lambda}_{\mathbf{2 8}}$ & $u(k-1)$ & $\mathbf{0 , 1 0 6 0 4}$ & $\mathbf{0}$ \\
\hline
\end{tabular}

Tabela 4. Validação do comportamento einâmico.

\begin{tabular}{cccc} 
Modelo & Regressor Retirado & $R M S E_{d}$ & $J_{d}$ \\
\hline $\boldsymbol{\Lambda}_{\mathbf{N 2}}$ & - & $\mathbf{0 , 1 7 8 5 9}$ & $\mathbf{0}$ \\
$\boldsymbol{\Lambda}_{\mathbf{2 1}}$ & $y(k-1)$ & $\mathbf{0 , 1 8 6 6 4}$ & $\mathbf{0 , 0 5 8 7 4}$ \\
$\Lambda_{22}$ & $y(k-2)$ & 0,19382 & 0,11330 \\
$\Lambda_{23}$ & $y(k-1)^{2}$ & 0,27474 & 0,87096 \\
$\Lambda_{24}$ & $y(k-2)^{2}$ & 0,24576 & 0,56955 \\
$\Lambda_{25}$ & $y(k-2) y(k-1)$ & 0,24609 & 0,57276 \\
$\Lambda_{26}$ & $C$ & 0,27671 & 0,89270 \\
$\boldsymbol{\Lambda}_{\mathbf{2 7}}$ & $u(k-1) y(k-1)$ & $\mathbf{0 , 2 5 9 7 3}$ & $\mathbf{0 , 7 1 0 7 1}$ \\
$\boldsymbol{\Lambda}_{\mathbf{2 8}}$ & $u(k-1)$ & $\mathbf{0 , 2 8 6 2 5}$ & $\mathbf{1}$ \\
\hline
\end{tabular}

Nesse segundo modelo nominal, ao retirar um dos regressores $C, u(k-1) y(k-1)$, principalmente, o $u(k-1)$, contribuirá significativamente na explicação do comportamento estático do sistema, mas em relação à explicação do comportamento dinâmico do sistema a retirada de qualquer regressor provoca uma piora. Desse modo, não existe nenhum modelo que melhore simultaneamente os erros estático e dinâmico, por isso, não serão estimados novos modelos. Nesse contexto, conforme a Figura 2, foi traçada a curva com as soluções eficientes. Esta apresentando também as soluções dominadas.

Conforme o que pode ser observado na Figura 2, o conjunto de soluções eficientes são formados pelos modelos: $\Lambda_{28}, \Lambda_{27}, \Lambda_{21}$ e $\Lambda_{\mathrm{N} 2}$. Os demais são soluções dominadas,

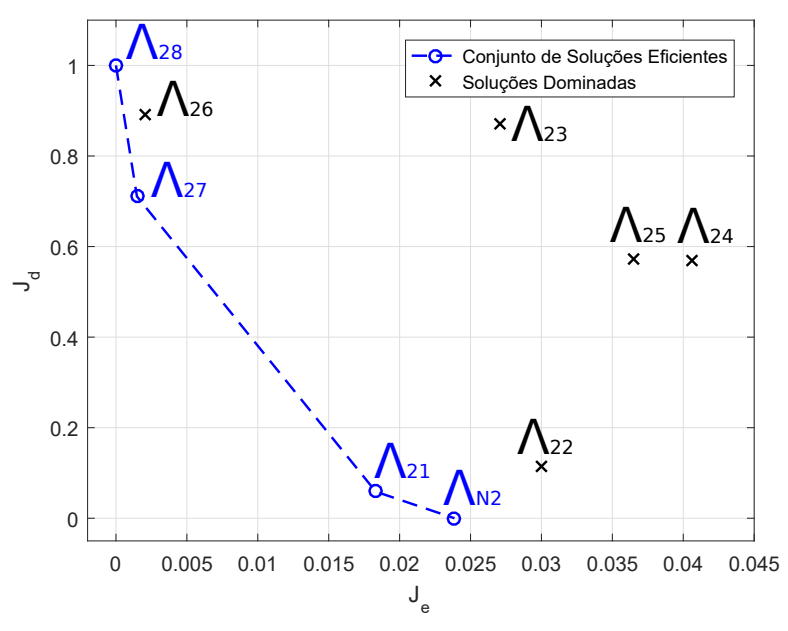

Figura 2. Conjunto de soluções eficientes e dominadas.

por apresentarem piora no comportamento estático e/ou dinâmico em comparação com as do conjunto eficiente. Dessa forma, apesar de o ponto de partida ser por meio de técnicas mono-objetivo, para otimização foi possível obter um conjunto de soluções eficientes que possuem elevada representatividade também do ponto de vista estático, tendo em vista mais de um objetivo.

A Figura 3 mostra as curvas estáticas do conversor CCCC buck obtido pela Equação 10 do modelo nominal $\left(\Lambda_{\mathrm{N}}\right)$ e dos modelos do conjunto eficiente. Estes apresentam uma melhora significativa no comportamento estático em comparação com o modelo inicial subótimo estimado via ERR e AIC. Vale salientar novamente que o sinal PRBS utilizado como entrada do sistema variou na faixa $2,2 \mathrm{~V}<$ $u<2,5 \mathrm{~V}$, sendo este o motivo do desajuste fora desta faixa entre os modelos e o sistema real. Já a Figura 4 apresenta as curvas com os dados de validação de simulação livre do comportamento dinâmico dos mesmos modelos.

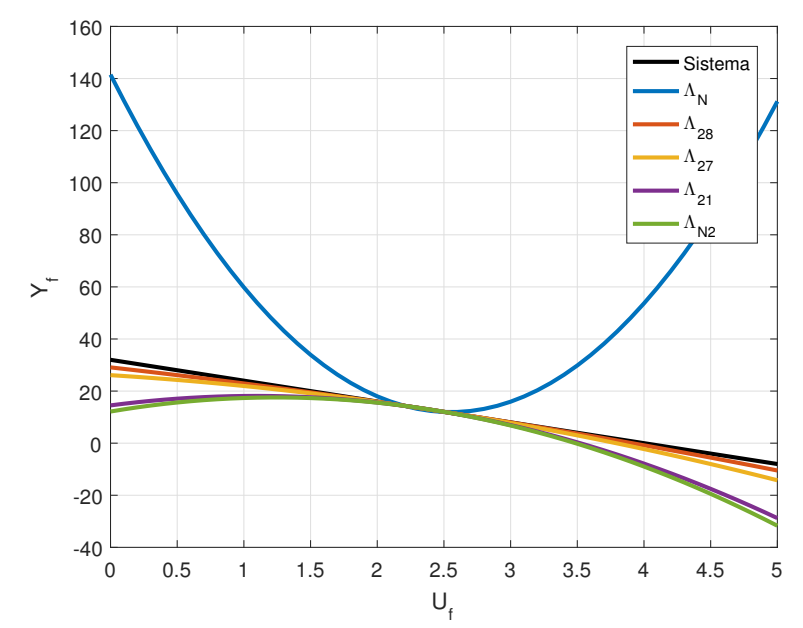

Figura 3. Curva estática do conversor CC-CC buck e dos modelos obtidos.

Com base nos resultados alcançados, partindo de um modelo subótimo $\left(\Lambda_{\mathrm{N}}\right)$ obtido via análise dinâmica, foi possível encontrar um modelo com dinâmica e estática melhoradas $\left(\Lambda_{\mathrm{N} 2}\right)$. Além disso, foi possível obter uma 


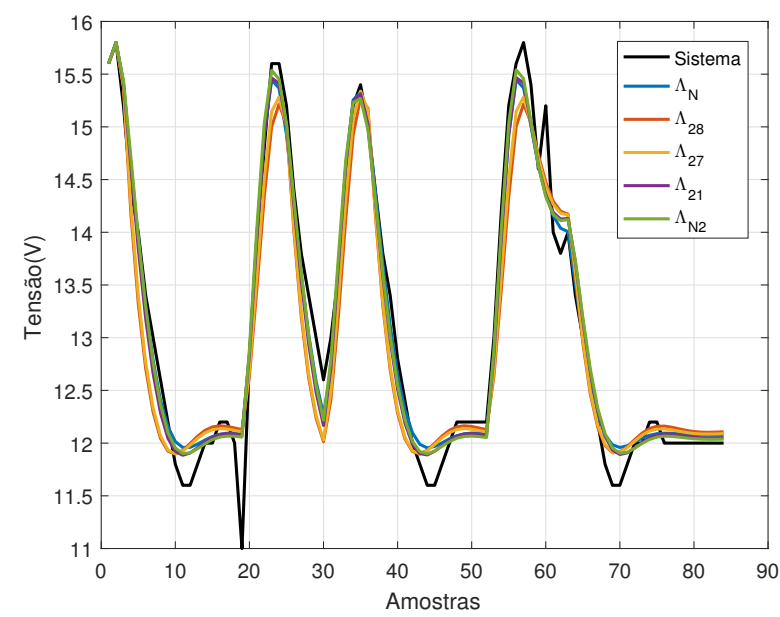

Figura 4. Curva dinâmica do conversor CC-CC buck e dos modelos obtidos.

família de modelos eficientes, sendo que a utilização destes depende da aplicação. É importante ressaltar que em todos os casos, os modelos obtidos são estruturalmente mais simples, o que diminui o custo computacional envolvido.

\section{CONCLUSÕES}

Nesse trabalho foi elaborada uma técnica para ser aplicada em modelos estimados via técnicas de caráter monoobjetivo como a ERR e AIC, que visa obter melhores modelos em vista dos comportamentos avaliados. A metodologia foi aplicada em um sistema composto por um Conversor CC-CC Buck, através da representação matemática NARMAX polinomial para identificá-lo. Com isso, verificou-se ser possível obter modelos que melhor representem os comportamentos analisados, como o estático e dinâmico do sistema, sendo obtido, assim, um conjunto de soluções eficiente.

Como proposta de trabalhos futuros, a técnica poderá ser aplicada em diferentes sistemas para comparação de resultados. Outra perspectiva consiste em avaliar se a estimativa de novos modelos com a inserção de regressores acarreta em alguma melhora no(s) comportamento(s) avaliado(s). Ademais, essa metodologia poderá ser aplicada em diferentes representações matemáticas.

\section{AGRADECIMENTOS}

Os autores agradecem a FAPEMIG, CNPq, INERGE, CAPES e a Universidade Federal de São Jõao del-Rei pelo apoio.

\section{REFERÊNCIAS}

Aguirre, L.A. (2007). Introdução à identificação de sistemas: técnicas lineares e não lineares aplicadas a sistemas reais. Editora UFMG. Belo Horizonte.

Aguirre, L.A., Donoso-Garcia, P.F., and Santos-Filho, R. (2000). Use of a priori information in the identification of global nonlinear models-a case study using a buck converter. IEEE Transactions on Circuits and Systems I: Fundamental Theory and Applications, 47(7), 10811085 .
Akaike, H. (1974). A new look at the statistical model identification. In Selected Papers of Hirotugu Akaike, 215-222. Springer.

Besharati, B., Luo, L., Azarm, S., and Kannan, P. (2006). Multi-objective single product robust optimization: An integrated design and marketing approach. Journal of Mechanical Design, 128(4), 884-892.

Billings, S.A. (1980). Identification of nonlinear systemsa survey. In IEE Proceedings D (Control Theory and Applications), volume 127, 272-285. IET.

Chen, S. and Billings, S.A. (1989). Representations of nonlinear systems: the narmax model. International journal of control, 49(3), 1013-1032.

Chiras, N., Evans, C., and Rees, D. (2001). Nonlinear gas turbine modeling using narmax structures. IEEE Transactions on Instrumentation and Measurement, 50(4), 893-898.

Genc, S. (2017). Parametric system identification using deep convolutional neural networks. In 2017 International Joint Conference on Neural Networks (IJCNN), 2112-2119. IEEE.

Ghosh, A. and Bhargava, P. (2018). Neural network forecasting for inflation in india: 2012-2017. Available at $S S R N$.

Gupta, S. and Kashyap, S. (2015). Forecasting inflation in g-7 countries: an application of artificial neural network. Foresight, 17(1), 63-73.

Huang, Y., Gao, L., Yi, Z., Tai, K., Kalita, P., Prapainainar, P., and Garg, A. (2018). An application of evolutionary system identification algorithm in modelling of energy production system. Measurement, 114, 122-131.

Irigoyen, E. and Miñano, G. (2013). A narx neural network model for enhancing cardiovascular rehabilitation therapies. Neurocomputing, 109, 9-15.

Korenberg, M., Billings, S., Liu, Y., and McIlroy, P. (1988). Orthogonal parameter estimation algorithm for non-linear stochastic systems. International Journal of Control, 48(1), 193-210.

Ljung, L. et al. (1987). Theory for the user. In System Identification. Prentice-hall, Inc.

Martins, S.A.M. and Aguirre, L.A. (2016). Sufficient conditions for rate-independent hysteresis in autoregressive identified models. Mechanical Systems and Signal Processing, 75, 607-617.

Martins, S.A.M., Nepomuceno, E.G., and Santos, M.F. (2012). Detecçao de estruturas de modelos narmax polinomiais: A taxa de reduçao de erro multi-objetivo (merr). In XIX Congresso Brasileiro de Automática, 18.

Nepomuceno, E.G. and Martins, S.A.M. (2016). A lower bound error for free-run simulation of the polynomial narmax. Systems Science \& Control Engineering, 4(1), 50-58.

Nepomuceno, E.G., Takahashi, R.H., and Aguirre, L.A. (2007). Multiobjective parameter estimation for nonlinear systems: affine information and least-squares formulation. International Journal of Control, 80(6), 863871.

Sukhai, A., Jones, A.P., Love, B.S., and Haynes, R. (2011). Temporal variations in road traffic fatalities in south africa. Accident Analysis \& Prevention, 43(1), 421-428. 